Bull. Soc. math. France

131 (3), 2003, p. $399-420$

\title{
DIMENSION OF WEAKLY EXPANDING POINTS FOR QUADRATIC MAPS
}

\author{
BY SAMUEL SENTI
}

\begin{abstract}
For the real quadratic map $P_{a}(x)=x^{2}+a$ and a given $\epsilon>0$ a point $x$ has good expansion properties if any interval containing $x$ also contains a neighborhood $J$ of $x$ with $\left.P_{a}^{n}\right|_{J}$ univalent, with bounded distortion and $B(0, \epsilon) \subseteq$ $P_{a}^{n}(J)$ for some $n \in \mathbb{N}$. The $\epsilon$-weakly expanding set is the set of points which do not have good expansion properties. Let $\alpha$ denote the negative fixed point and $M$ the first return time of the critical orbit to $[\alpha,-\alpha]$. We show there is a set $\mathcal{R}$ of parameters with positive Lebesgue measure for which the Hausdorff dimension of the $\epsilon$-weakly expanding set is bounded above and below by $\log _{2} M / M+\mathcal{O}\left(\log _{2} \log _{2} M / M\right)$ for $\epsilon$ close to $|\alpha|$. For arbitrary $\epsilon \leq|\alpha|$ the dimension is of the order of $\mathcal{O}\left(\log _{2}\left|\log _{2} \epsilon\right| /\left|\log _{2} \epsilon\right|\right)$. Constants depend only on $M$. The Folklore Theorem then implies the existence of an absolutely continuous invariant probability measure for $P_{a}$ with $a \in \mathcal{R}$ (Jakobson's Theorem).
\end{abstract}

Texte reçu le 11 février 2002, révisé le 15 juillet 2002, accepté le 16 septembre 2002

Samuel Senti, Department of Mathematics, Penn State University, University Park, PA, 16802 (USA) - E-mail : senti@math.psu.edu • Url:http://www.math.psu.edu/senti 2000 Mathematics Subject Classification. - 37E05, 37D25, 37D45, 37C45.

Key words and phrases. - Quadratic map, Jakobson's theorem, Hausdorff dimension, Markov partition, Bernoulli map, induced expansion, absolutely continuous invariant probability measure. 
RÉSUMÉ (Dimension des points faiblement dilatants pour l'application quadratique) Pour l'application quadratique réelle $P_{a}(x)=x^{2}+a$ et un $\epsilon>0$ donné, un point $x$ a de bonnes propriétés de dilatation si tout intervale contenant $x$ contient également un voisinage $J$ de $x$ avec $\left.P_{a}^{n}\right|_{J}$ univalent, avec distortion bornée et $B(0, \epsilon) \subseteq P_{a}^{n}(J)$ pour un $n \in \mathbb{N}$. L'ensemble $\epsilon$-faiblement dilatant est l'ensemble des points qui n'ont pas de bonnes propriétes de dilatation. Notons $\alpha$ le point fixe négatif et $M$ le temps de premier retour de l'orbite critique dans $[\alpha,-\alpha]$. Nous prouvons l'existence d'un ensemble $\mathcal{R}$ de paramètres de mesure de Lebesgue positive pour lesquels la dimension de Hausdorff de l'ensemble $\epsilon$-faiblement dilatant est bornée supérieurement et inférieurement par $\log _{2} M / M+\mathcal{O}\left(\log _{2} \log _{2} M / M\right)$ si $\epsilon$ est proche de $|\alpha|$. Pour $\epsilon \leq|\alpha|$ quelconque la dimension est de l'ordre de $\mathcal{O}\left(\log _{2}\left|\log _{2} \epsilon\right| /\left|\log _{2} \epsilon\right|\right)$. Les constantes ne dependent que de $M$. Le théorème du Folklore implique alors l'existence d'une mesure de probabilité absolument continue et invariante par $P_{a}$ pour $a \in \mathcal{R}$ (théorème de Jakobson).

\section{Introduction}

The 1-parameter family of real quadratic maps $P_{a}(x)=x^{2}+a$ is a simple model of nonlinear dynamics exhibiting surprisingly rich dynamical structure and giving rise to interesting and difficult questions. For instance, the existence of a $P_{a}$-invariant probability measure which is absolutely continuous with respect to the Lebesgue measure Leb, a so-called a.c.i.p. , for a set of parameters of positive Lebesgue measure was first proved by Jakobson in [9] (see also [4], [14], [16], [10], [17], [15]).

Much is known about the real quadratic family, including a positive answer for the real Fatou conjecture (see [8], [11]) as well as for its generalization to real analytic families of maps with one non-flat critical point and negative Schwarzian derivative, so-called $S$-unimodal families [1]. Avila and Moreira have also shown that almost every non-regular parameter (no periodic hyperbolic attractor) satisfies the Collet-Eckmann condition for the quadratic map [3] as well as for a residual set of analytical $S$-unimodal families with a quadratic critical point [2]. However, many questions remain for $S$-unimodal maps, Hénon maps (see [5]) and complex quadratic maps. The real quadratic map enjoys a particular status as it serves as a model to help understand these problems.

A common technique to build an a.c.i.p. for an interval map $f$ is to look for expansion for some iterate of the original map on a properly restricted domain $J_{i}$. The map $\left.T\right|_{J_{i}}:=\left.f^{n_{i}}\right|_{J_{i}}$ is called the induced map. The Folklore Theorem (see for instance [12], [6], [13]) asserts the existence of a $T$-invariant a.c.i.p. $\nu$ provided there exists $\epsilon>0$ and a $T$-invariant interval $A$ containing the critical point satisfying the following three conditions: a) $A$ is the countable union, modulo sets of zero Lebesgue measure, of intervals $J_{i}$ with disjoint interiors, b) $T$ restricted to each $J_{i}$ is a diffeomorphism with uniformly bounded distortion and c) $\operatorname{Leb}\left(T\left(J_{i}\right)\right) \geq \epsilon$. If additionally the summability condition 
$\sum n_{i} \nu\left(J_{i}\right)<\infty$ holds, then there exists an a.c.i.p. $\mu$ which is invariant by the original map $f$.

Let $B(0, \epsilon)$ denote the ball of radius $\epsilon$ centered at 0 . For the quadratic family $P_{a}$ and a given $\epsilon>0$, we will say that a point $x$ has good expansion properties if any interval containing $x$ also contains a neighborhood $J$ of $x$ with $\left.P_{a}^{n}\right|_{J}$ univalent with bounded distortion and $B(0, \epsilon) \subseteq P_{a}^{n}(J)$ for some iterate $n \in \mathbb{N}$. We call points which do not have good expansion properties $\epsilon$ weakly expanding. For such points there are only a finite number of iterates for which one can find a neighborhood $J$ of $x$ with $\left.P_{a}^{n}\right|_{J}$ univalent with bounded distortion and $B(0, \epsilon) \subseteq P_{a}^{n}(J)$

In this work, we estimate the Hausdorff dimension of the $\epsilon$-weakly expanding set for the quadratic map for a set of parameters of positive Lebesgue measure. More precisely if $\alpha$ is the negative fixed point and

$$
M:=\min \left\{n \in \mathbb{N} ;\left|P_{a}^{n}(0)\right|<|\alpha|\right\}
$$

is the first return time of the critical orbit to $[\alpha,-\alpha]$, we prove the following:

Main Theorem. - For a set of parameters $\mathcal{R}$ with

$$
\lim _{\delta \rightarrow 0} \frac{\operatorname{Leb}(\mathcal{R} \cap[-2,-2+\delta))}{\delta}=1
$$

the Hausdorff dimension of the $\epsilon$-weakly expanding set $\mathcal{E}_{\epsilon}$ is

$$
\operatorname{dim}_{H}(\mathcal{E} \epsilon)=\frac{\log _{2} M}{M}\left(1+\mathcal{O}\left(\frac{\log _{2} \log _{2} M}{\log _{2} M}\right)\right)
$$

for $c 2^{-M}<\epsilon \leq|\alpha|$ and a constant $c>0$. For $0<\epsilon \leq|\alpha|$ arbitrary, we have

$$
\operatorname{dim}_{H}\left(\mathcal{E}_{\epsilon}\right)=\mathcal{O}\left(\frac{\log _{2}\left|\log _{2} \epsilon\right|}{\left|\log _{2} \epsilon\right|}\right) .
$$

Constants depend only on the return time $M$.

For parameters in $\mathcal{R}$ close to -2 the return time $M$ is large, so points with good expansion properties have full Lebesgue measure. For $\epsilon=|\alpha|$ the Folklore Theorem applies and Jakobson's Theorem follows as a Corollary once we prove the summability condition.

Corollary (Jakobson [9]). - For the real quadratic map $P_{a}$, there exists a probability measure which is invariant by $P_{a}$ and absolutely continuous with respect to the Lebesgue measure for a set of parameters $a \in \mathcal{R}$ of positive Lebesgue measure. 
Structure of the paper. - We consider the neighborhood $A:=[\alpha,-\alpha]$ of the critical point in Section 2. We build a partition of $A$ consisting of those preimages of $A$ on which the induced map $T$ can be extended as a diffeomorphism to a given larger domain containing $A$. Such extendibility insures uniform bounded distortion by Koebe's distortion property and the image by $T$ of the partition elements obviously contain the ball of radius $\epsilon<|\alpha|$ centered at 0 .

In Section 3 we identify the sets that contain $\epsilon$-weakly expanding points with $\epsilon=\alpha$.

In Section 4 we introduce Yoccoz's strongly regular parameter conditions [17] and show that for these parameters the $\alpha$-weakly expanding set is the attractor of an iterated function system (IFS) with countably many generators.

In Section 5, we obtain derivative estimates for the generators of the IFS by viewing the quadratic map as a perturbation of the Chebyshev polynomial $x^{2}-2$ for parameters close to -2 (large $M$ ). We also prove that strongly regular parameters satisfy the Collet-Eckmann condition.

In Section 6 we bound the Hausdorff dimension of the $\alpha$-weakly expanding set. The lower bound is obtained by approximating this set by the attractor of an IFS with finitely many generators and estimating the dimension of the approximation (which obviously contains the $\alpha$-weakly expanding set). If the number of generators of the approximation is large enough, the difference between both dimensions lies within the error bounds produced by the derivative estimates giving us an upper bound. The complement of the partition of $A$ coincides with the $\epsilon$-weakly expanding set with $c 2^{-M}<\epsilon \leq|\alpha|$ for some constant $c>0$. We prove a measure estimate which implies both the summability condition and the full Lebesgue measure of the partition of $A$. The Folklore Theorem together with the positive Lebesgue measure of strongly regular parameters (see [17], [15]) then implies Jakobson's Theorem [9].

In Section 7 we consider the $\epsilon$-weakly expanding set for general $\epsilon$. We generalize the construction introduced in the first part, showing that the $\epsilon$-weakly expanding set is the attractor of an IFS with countably many generators. In this case only very crude derivative estimates are necessary. We bound the number of contractions for which the same estimates are used and proceed similarly as in the first part to estimate the dimension of the $\epsilon$-weakly expanding set.

Acknowledgments. - The author would like to thank J.-C. Yoccoz for his uncounted time, support and inspiration. Thanks also to M. Jakobson for his interest and support, to the Université d'Orsay, where this research was conducted, and to the Penn State University, where this article was written.

\section{Definitions}

For the real quadratic map

$$
P_{a}(x)=x^{2}+a
$$

TOME $131-2003-\mathrm{N}^{\mathrm{O}} 3$ 
with parameters $-\frac{3}{4}>a \in \mathbb{R}$ denote the fixed points by $\beta>0$ and $\alpha<0$. For $n \in \mathbb{N}$, set $\alpha^{0}:=\alpha$ and define inductively $\alpha^{n}$ as the unique negative point for which

$$
P_{a}\left(\alpha^{n}\right)=-\alpha^{n-1} .
$$

If $a<\alpha^{n-1}$ define inductively $\widetilde{\alpha}^{n}$ as the unique negative point for which

$$
P_{a}\left(\widetilde{\alpha}^{n}\right)=\alpha^{n-1} \text {. }
$$

Choose $M \in \mathbb{N}$ large and consider parameters for which $P_{a}(0) \in\left(\alpha^{M-1}, \alpha^{M-2}\right)$. Let

$$
A:=[\alpha,-\alpha] \subseteq\left[\alpha^{1},-\alpha^{1}\right]=: \widehat{A} .
$$

Set $C_{1}^{+}=\left[\alpha^{1}, \alpha\right]$ and

$$
C_{n}^{+}:=\left[\widetilde{\alpha}^{n-1}, \widetilde{\alpha}^{n}\right] \text { for } 2 \leq n \leq M-2 .
$$

Also write $C_{n}^{-}=-C_{n}^{+}$for the interval symmetric to $C_{n}^{+}$.

Definition 2.1. - An interval $J \subseteq[-\beta, \beta]$ is regular of order $n \geq 0$ if there exists a neighborhood $\widehat{J}$ of $J$ such that the restriction of $P_{a}^{n}$ to $\widehat{J}$ is a diffeomorphism onto $\widehat{A}$ and $P_{a}^{n}(J)=A$. We denote the order of a regular interval by $\operatorname{ord}(J)$. Denote

$$
\mathcal{J}:=\left\{J \subsetneq A \text { regular } ; \forall J^{\prime} \text { regular with } \operatorname{int}\left(J^{\prime}\right) \cap \operatorname{int}(J) \neq \varnothing \Rightarrow J^{\prime} \subseteq J\right\} .
$$

Elements of $\mathcal{J}$ are maximal with respect to inclusion.

Proposition 2.2. - a) Regular intervals are nested or have disjoint interiors.

b) $\left[\alpha^{n}, \alpha^{n-1}\right]$ is regular and maximal for all $n \geq 1 . C_{n}^{ \pm} \in \mathcal{J}$ are regular and maximal for $2 \leq n \leq M-2$. As $\lim _{n \rightarrow \alpha^{n}}=-\beta$, regular intervals cover $[-\beta, \alpha]$. As the multiplier of $\alpha$ is negative, there are no maximal regular intervals of order 1 in $[\alpha,-\alpha]\left(\left[\alpha^{1}, \alpha\right]\right.$ and $-\left[\alpha^{1}, \alpha\right]$ are the only regular intervals of order 1).

c) The image $P_{a}(J)$ of a regular interval $J$ of order $n \geq 1$ is regular of order $n-1$ and $\widehat{P_{a}(J)}=P_{a}(\widehat{J}) \subseteq(a, \beta)$. Conversely, if $J$ is (maximal) regular of order $n \geq 0$, and if $\widehat{J} \subseteq(a, \beta)$, then both components of $P_{a}^{-1}(J)$ are (maximal) regular of order $n+1$.

d) For each regular interval $J$ there are two adjacent regular intervals $J_{i}$, $i=1,2$, with $\operatorname{int}\left(J_{i}\right) \cap \operatorname{int}(J)=\varnothing$ and $\operatorname{ord}\left(J_{i}\right)=\operatorname{ord}(J)+3$. If $J \in \mathcal{J}$ there are two adjacent maximal regular intervals $J_{i}^{\prime}$ with $\operatorname{int}\left(J_{i}^{\prime}\right) \cap \operatorname{int}(J)=\varnothing$ and ord $J_{i}^{\prime}-\operatorname{ord} J=\{ \pm 1, \pm 3\}$.

Definition 2.3. - For a regular interval $J$ denote by $\mathcal{G}_{J}: \widehat{A} \rightarrow \widehat{J}$ the local inverse to $\left.P_{a}^{\operatorname{ord}(J)}\right|_{J}$. Also

$$
W_{n}:=\bigcup_{\substack{J \in \mathcal{J} \\ 2 \leq \operatorname{ord}(J) \leq n}} \operatorname{int}(J), \quad W=\bigcup_{n \geq 2} W_{n}
$$

BULletin DE LA SOCIÉtÉ MATHÉMATIQUE DE FRANCE 


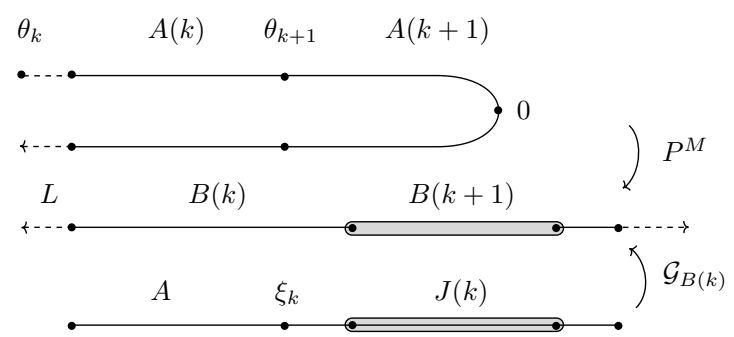

Figure 1.

For $x \in \operatorname{int}(J), J \in \mathcal{J}$, the return time is the function $N: W \rightarrow \mathbb{N}$ given by

$$
N(x):=\operatorname{ord}(J)
$$

and the induced map is the function $T: W \rightarrow \operatorname{int}(A)$ given by

$$
T(x):=P_{a}^{o r d(J)}(x) .
$$

Accept the abuse of notation $T(0):=P^{M}(0)$.

Definition 2.4. - A gap of order $n$ is a connected component of $A \backslash \bar{W}_{n}$. The set of all gaps of order $n$ is denoted by $\varepsilon(n)$. The primary $(\alpha-)$ weakly expanding set

$$
\widetilde{\mathcal{E}}:=\bigcap_{n \geq 1} A \backslash \bar{W}_{n}=\bigcap_{n \in \mathbb{N}} \varepsilon(n)
$$

is the set of non isolated points of $A \backslash W$. The $(\alpha-)$ weakly expanding set is

$$
\mathcal{E}=\bigcup_{k \geq 0} P_{a}^{-k}(\widetilde{\mathcal{E}}) .
$$

We have $\operatorname{dim}_{H}(\mathcal{E})=\operatorname{dim}_{H}(\widetilde{\mathcal{E}})$.

For $2 \leq n \leq M-2, \varepsilon(n)=\left(\widetilde{\alpha}^{n},-\widetilde{\alpha}^{n}\right)$ since $C_{n}^{ \pm} \in \mathcal{J}$. As $\left[\widetilde{\alpha}^{M-2}, \widetilde{\alpha}^{M-1}\right]$ is not regular, $\mathcal{J}$ has no elements of order either $M-1$ or $M$ so

$$
\varepsilon(M-1)=\varepsilon(M)=\left(\widetilde{\alpha}^{M-2},-\widetilde{\alpha}^{M-2}\right) .
$$

Hence $\varepsilon(n) \subseteq\left(\widetilde{\alpha}^{M-2},-\widetilde{\alpha}^{M-2}\right)$ for $n \geq M-2$.

In the following assume: $T(0) \in \bigcap_{n \geq 1} \operatorname{Dom}\left(T^{n}\right)$.

Definition 2.5. - For $k \geq 1$, let $J(k) \in \mathcal{J}$ with $T^{k}(0) \in \operatorname{int}(J(k))$. Set

$$
\begin{aligned}
& n_{0}:=0, n_{k}:=N\left(T^{k}(0)\right), \\
& N_{0}:=M, \quad N_{k}=N_{k-1}+n_{k-1}=M+\sum_{0 \leq i \leq k-1} n_{i} .
\end{aligned}
$$

TOME $131-2003-\mathrm{N}^{\mathrm{O}} 3$ 
Define a nested sequence of regular intervals $B(k)$ containing $P^{M}(0)$ by

$$
B(1):=A, \quad B(k)=\mathcal{G}_{B(k)}(A):=\mathcal{G}_{J(1)} \circ \cdots \circ \mathcal{G}_{J(k-1)}(A)
$$

(see Fig. 1). Let $\zeta \in\left[\widetilde{\alpha}^{M-2}, \widetilde{\alpha}^{M-1}\right]$ be the point with $P_{a}^{M-1}(\zeta)=0$ and let $Q:=\left.P_{a}^{M}\right|_{(\zeta,-\zeta)}$. Then define

$$
A(k):=Q^{-1}(B(k)) .
$$

Let $L$ be the maximal (with respect to inclusion) regular interval adjacent to $B(k)$ and contained in $P_{a}^{M}((\zeta,-\zeta)) \cap(\widehat{B}(k) \backslash$ int $B(k))$. Set

$$
\widehat{A}(k):=\left[\theta_{k},-\theta_{k}\right]:=Q^{-1}(B(k) \cup L) .
$$

If $P_{a}^{M}((\zeta,-\zeta)) \cap(\widehat{B}(k) \backslash$ int $B(k))$ is regular and $A(k) \neq A(k+1)$, then by Proposition $2.2 \widehat{A}(k) \backslash \operatorname{int}(A(k))$ is maximal and regular of order $N_{k}^{\prime}=N_{k}+1$. Otherwise, $\theta_{k}$ is the left endpoint of a maximal regular interval of order $N_{k}^{\prime}=N_{k}+3$. Also set $\xi_{0}=\alpha^{1}$ and $\xi_{k}=P_{a}^{N_{k}}\left(\theta_{k+1}\right)$.

Let us make the following orientation assumption:

$$
P^{N_{k}}(x) \leq P^{N_{k}}(0) \text { for all } x \in \widehat{A}(k) .
$$

The reversed orientation case is similar. Write

$$
\mathcal{K}_{k}:=\left\{I \text { regular } ; P_{a}^{N_{k}}(0) \in \widehat{I} \text { and } I \nsubseteq\left[\xi_{k}, \beta\right]\right\} .
$$

Proposition 2.6. - For all $I, I^{\prime} \in \mathcal{K}_{k}$ we have $\operatorname{int}(I) \cap \operatorname{int}\left(I^{\prime}\right)=\varnothing$, except for the two regular intervals adjacent to $J(k)$ of order $\operatorname{ord}(I):=p=n_{k}-3$ and $\operatorname{ord}\left(I^{\prime}\right)=n_{k}-1$. Also $\operatorname{ord}(I) \neq \operatorname{ord}\left(I^{\prime}\right)$ and $J(k) \subset \widehat{I}$.

If $n_{k} \leq M-2$ then $\mathcal{K}_{k}=\left\{C_{n_{k}-1}^{+}\right\}$if $0 \notin P_{a}^{N_{k}}(A(k))$ and $\mathcal{K}_{k}=\varnothing$ otherwise.

If $n_{k} \geq M+1$ then $M-2 \leq p<n_{k}$

Proof. - Assume first $\xi_{k} \notin \operatorname{int}(I)$. Regular intervals are nested or disjoint. If $I^{\prime} \subset I$ then $p^{\prime}:=\operatorname{ord}\left(I^{\prime}\right) \geq p+2$. Let $I^{\prime \prime}$ denote both regular intervals of order $p+3$ adjacent to but not contained in $I$. Then $\widehat{I}^{\prime} \subseteq I \cup I^{\prime \prime}$ or else there would be a point $x \in \operatorname{int}\left(\widehat{I}^{\prime} \backslash I^{\prime}\right)$ with $P_{a}^{p+3}(x)=-\alpha$. As $J(k) \in \mathcal{J}$ and $\xi_{k} \notin \operatorname{int}(I)$ we have $J(k) \nsubseteq I^{\prime \prime}$ so $\widehat{I}^{\prime} \cap J(k)=\varnothing$ and $I^{\prime} \notin \mathcal{K}_{k}$. So $I, I^{\prime} \in \mathcal{K}_{k}$ have disjoint interiors and for $I^{\prime}$ between $I$ and $J(k)$ we have $I^{\prime} \subseteq \widehat{I} \backslash I$ as $\widehat{I} \cap \operatorname{int}(J(k)) \neq \varnothing$ so $p+3 \leq p^{\prime}$.

Now assume $\xi_{k} \in \operatorname{int}(I)$. The component of $\widehat{J}(k) \backslash \operatorname{int}(J(k))$ containing $\xi_{k}$ is regular, or else, by Definition 2.5 and Proposition $2.2, \xi_{k}$ is the endpoint of a maximal regular interval. This contradicts $\xi_{k} \in \operatorname{int}(I)$ with $I$ regular. Regular intervals are nested or have disjoint interiors so $I$ and $J(k)$ are adjacent and $J(k) \in \mathcal{J}$ implies $p=n_{k}-1$ or $n_{k}-3$. 
For $n_{k} \leq M-2$, if $0 \notin P_{a}^{N_{k}}(A(k))$ then $J(k)=C_{n_{k}}^{+}$and $\widehat{I} \cap \operatorname{int}(J(k))=\varnothing$ for all regular $I \nsubseteq\left[\xi_{k}, \beta\right]$ except $I=C_{n_{k}-1}^{+}$for which $J(k) \subseteq \widehat{I}$. If $0 \in P_{a}^{N_{k}}(A(k))$ then $J(k)=C_{n_{k}}^{-}$and $\widehat{I} \cap \operatorname{int}(J(k))=\varnothing$ for all regular $I \nsubseteq\left[\xi_{k}, \beta\right]$.

For $n_{k} \geq M+1$, no interval of order $<M-2$ intersects int $(J(k))$. Assume $p \geq n_{k}$. Then $\xi_{k} \notin \operatorname{int}(I)$ and $\widehat{I} \cap \operatorname{int}(J(k)) \neq \varnothing$ so one of the endpoints of $J(k)$ is contained in $\widehat{I} \backslash I$ and $p+3 \leq n_{k}$ contradicting the assumption. So $M-2 \leq p<n_{k}$. Also if $J(k) \nsubseteq \widehat{I}$, then $\operatorname{int}(J(k))$ would contain an endpoint of $\widehat{I}$ as $\widehat{I} \cap \operatorname{int}(J(k)) \neq \varnothing$. But then $p+1 \geq n_{k}+2$ contradicting $p<n_{k}$.

\section{Classification of the gaps}

For $M+3 \leq n \leq N_{\ell+1}$, let $\bar{\ell}$ be the largest integer for which $N_{\bar{\ell}}^{\prime} \leq n$ with $N_{\bar{\ell}}^{\prime}$ from Definition 2.5. Define

$$
\varepsilon(n, 0)=\{J \in \varepsilon(n) ; J \subseteq \widehat{A} \backslash \operatorname{int}(\widehat{A}(1))\}, \quad \varepsilon_{c}(n)=\{J \in \varepsilon(n) ; J \subseteq \widehat{A}(\bar{\ell})\}
$$

and for $1 \leq k<\bar{\ell}$

$$
\varepsilon(n, k)=\{J \in \varepsilon(n) ; J \subseteq \widehat{A}(k) \backslash \operatorname{int}(\widehat{A}(k+1))\} .
$$

Proposition 3.1. - A gap of $\varepsilon(n, k)$ is one of both pre-images

- either by $Q^{-1} \circ \mathcal{G}_{B(k)}$ of a gap of $\varepsilon\left(n-N_{k}\right) \cap\left[\alpha, \xi_{k}\right]$;

- or by $Q^{-1} \circ \mathcal{G}_{B(k)} \circ \mathcal{G}_{I}$ of a gap of $\varepsilon\left(n-N_{k}-\operatorname{ord}(I)\right)$ where $I \in \mathcal{K}_{k}$.

Proof. - Let $J \in \mathcal{J}$ with $J \subseteq \widehat{A}(k) \backslash \operatorname{int} \widehat{A}(k+1)$. By Proposition $2.2 P_{a}^{N_{k}}(J) \subseteq$ $\left[\alpha^{1}, \xi_{k}\right]$ is a regular interval of order $\operatorname{ord}\left(P_{a}^{N_{k}}(J)\right)=\operatorname{ord}(J)-N_{k}$. If $P_{a}^{N_{k}}(J)$ is not maximal there exists $I \in \mathcal{K}_{k}$ with $P_{a}^{N_{k}}(J) \subseteq I$. Then $P_{a}^{N_{k}+\operatorname{ord}(I)}(J) \in \mathcal{J}$, unless $I$ is the regular interval adjacent to $J(k)$ with $\operatorname{ord}(I)=n_{k}-3$ and $P_{a}^{N_{k}}(J) \subsetneq I^{\prime}$ for $I^{\prime} \in \mathcal{K}_{k}$ with $\operatorname{ord}\left(I^{\prime}\right)=n_{k}-1$. Then $P_{a}^{N_{k}+\operatorname{ord}\left(I^{\prime}\right)}(J) \in \mathcal{J}$.

Conversely, for any maximal regular interval $I \subseteq\left[\alpha^{1}, \xi_{k}\right]$, we have $\widehat{I} \subseteq(a, \beta)$ and by Proposition 2.2 both components of $P_{a}^{-1}(I)$ are regular and maximal with respect to inclusion. If $I \notin \mathcal{K}_{k}$, the same argument yields that both pre-images $Q^{-1} \circ \mathcal{G}_{B(k)}(I)$ contained in $\widehat{A}(k)$ are maximal regular intervals of order $\operatorname{ord}(I)+N_{k}$. If $I \in \mathcal{K}_{k}$ consider the images $\mathcal{G}_{I}(J)$ of maximal regular intervals $J$. Passing to the complementary set yields the proposition.

Corollary 3.2. - A gap of $\varepsilon(n, 0)$ is contained in either $\left[\widetilde{\alpha}^{M-1}, \widetilde{\alpha}^{M-2}\right]$ or its symmetric and is a pre-image by $P_{a}^{-(M-1)}$ of a gap of $\varepsilon(n-M+1)$.

Definition 3.3. - Set $D_{0}:=\left[\widetilde{\alpha}^{M-2},-\widetilde{\alpha}^{M-2}\right]$ and $D_{k}=D_{0} \cap\left[\alpha, \xi_{k}\right]$. Denote:

- $\mathcal{G}_{0}^{ \pm}$the restriction to $D_{0}$ of the positive/negative branch of the inverse map to $\left.P_{a}^{M-1}\right|_{\left[\widetilde{\alpha}^{M-2}, \widetilde{\alpha}^{M-1}\right]}$.

TOME $131-2003-\mathrm{N}^{\mathrm{O}} 3$ 
- $\mathcal{G}_{k}^{ \pm}$the restriction to $D_{k} \neq \varnothing$ of the positive/negative branch of the inverse map to $Q^{-1} \circ \mathcal{G}_{B(k)}$.

- $\mathcal{G}_{k, I}^{ \pm}$the restriction to $D_{0}$ of the positive/negative branch of the inverse map to $Q^{-1} \circ \mathcal{G}_{B(k)} \circ \mathcal{G}_{I}$ for $I \in \mathcal{K}_{k} \neq \varnothing$.

No mapping is to be defined if $D_{k}=\varnothing$ or $\mathcal{K}_{k}=\varnothing$. Denote $\mathcal{G}_{0, I}^{ \pm}:=\mathcal{G}_{0}^{ \pm}$and $\Gamma=\left\{\mathcal{G}_{k}^{\epsilon} ; \epsilon= \pm, k \geq 0, D_{k} \cap \widetilde{\mathcal{E}} \neq \varnothing\right\} \cup\left\{\mathcal{G}_{k, I}^{\epsilon} ; \epsilon= \pm, k \geq 0, I \in \mathcal{K}_{k} \neq \varnothing\right\}$.

\section{Strongly regular parameters}

Fix a large $\bar{M} \in \mathbb{N}$ with $\log ^{\kappa} M<\bar{M}<\frac{2}{3} M$ for some $\kappa>1$ and $\rho \in \mathbb{R}$ with $\bar{M} 2^{-\bar{M}} \ll \rho \ll 1$.

Definition 4.1 (see [17]). - A parameter $a$ is strongly regular if

a) $T(0) \in \bigcap_{n \geq 1} \operatorname{Dom}\left(T^{n}\right)$,

b) $\sum_{\substack{1 \leq i \leq k, N\left(T^{i}(0)\right) \geq \bar{M}}} N\left(T^{i}(0)\right)<\rho k$ for all $k \in \mathbb{N}$.

Proposition 4.2. - For a strongly regular parameter and $q=\left[\rho^{-1} \bar{M}\right]$ the integer part of $\rho^{-1} \bar{M}$,

$$
N\left(T^{k}(0)\right)<\bar{M} \text { for } k \leq q, \quad N\left(T^{k}(0)\right)<\rho k \text { for } q<k .
$$

COROLLARY 4.3. - Let $F$ be the attractor of the iterated function system generated by the $\mathcal{G}_{k}, \mathcal{G}_{k, I} \in \Gamma$ with $k \leq q$. For strongly regular parameters $F \subseteq \tilde{\mathcal{E}}$ and $\widetilde{\mathcal{E}}$ is invariant by all $\mathcal{G}_{k}, \mathcal{G}_{k, I} \in \Gamma$ with $k \leq q$.

Proof. - Follows from Definition 2.4 and from Proposition 3.1 as $\widetilde{\mathcal{E}} \subset D_{0}=D_{k}$ for strongly regular parameters.

\section{Derivative estimates}

Proposition 5.1. - Let $x \in D_{k}$. For strongly regular parameters, there exists a constant $c>0$ with

1) For $0 \leq k \leq q$ :

$$
c^{-1} 2^{-\frac{1}{2}\left(M+N_{k}-n_{k}\right)} \leq\left|D \mathcal{G}_{k}^{ \pm}(x)\right| \leq c 2^{-\frac{1}{2}\left(M+N_{k}-n_{k}\right)}
$$

2) For $k>q$ and $x \in D_{k}$ :

$$
c^{-1} 2^{-\frac{1}{2}\left(M+N_{k}\right)-\rho k} \leq\left|D \mathcal{G}_{k}^{ \pm}(x)\right| \leq c 2^{-\frac{1}{2}\left(M+N_{k}\right)+n_{k}+\rho k} .
$$

3) For $0 \leq k \leq q$ :

$$
c^{-1} 2^{-\frac{1}{2}\left(M+N_{k+1}\right)} \leq\left|D \mathcal{G}_{k, I}^{ \pm}(x)\right| \leq c 2^{-\frac{1}{2}\left(M+N_{k+1}\right)}
$$

BULletin DE LA SOCIÉtÉ MATHÉMATIQUE DE FRANCE 
4) For $k>q$ :

$$
c^{-1} 2^{-\frac{1}{2}\left(M+N_{k}+\operatorname{ord}(I)\right)-2 \rho k} \leq\left|D \mathcal{G}_{k, I}^{ \pm}(x)\right| \leq c 2^{-\frac{1}{2}\left(M+N_{k}+\operatorname{ord}(I)\right)+2 \rho k}
$$

LEMMA 5.2. - For parameters with $P_{a}(0) \in\left(\alpha^{M-1}, \alpha^{M-2}\right)$, there exists a constant $c>0$ with

$$
\begin{array}{ll}
c^{-1} 4^{-n} \leq \alpha^{n}-P_{a}(0) \leq c 4^{-n} & \text { for } 0 \leq n<M-2 \\
c^{-1} 2^{-n} \leq-\widetilde{\alpha}^{n} \leq c 2^{-n} & \text { for } 0<n \leq M-2, \\
c^{-1} 4^{-M} \leq \beta+P_{a}(0) \leq c 4^{-M} . &
\end{array}
$$

Proof. - For these parameters, consider $P_{a}$ as a perturbation of the Chebyshev polynomial. The estimates follow. See [15]

Lemma 5.3. - Write $h(x):=\left(\beta^{2}-x^{2}\right)^{-\frac{1}{2}}$ for $|x|<\beta$. Then

$$
\left|D P_{a}^{n}(x)\right|=2^{n} \frac{h(x)}{h\left(P_{a}^{n}(x)\right)} \prod_{i=0}^{n-1}\left(1+\frac{\beta+a}{\left(P_{a}^{i}(x)\right)^{2}}\right)^{-\frac{1}{2}} .
$$

Proof. - Follows by induction using $\beta^{2}+a=\beta$.

LEMMA 5.4. - For strongly regular parameters, there is a constant $c>0$ with

1) $\left|\log _{2}\right| D P_{a}^{n}(x) \frac{h\left(P_{a}^{n}(x)\right)}{h(x)}|-n| \leq c n 4^{-M}$ for $1 \leq n$ and $x \in\left[\alpha^{n}, \alpha^{n-1}\right]$.

2) $\left|\log _{2}\right| D \mathcal{G}_{C_{n}^{ \pm}}(x) \frac{h\left(\mathcal{G}_{C_{n}^{ \pm}}(x)\right)}{h(x)}|+n| \leq c 4^{n-M}$ for $1 \leq n \leq M-2$ and $x \in A$.

3) $4^{- \text {ord } J} \leq\left|D \mathcal{G}_{J}(x)\right| \leq c$ for all regular $J$ and $x \in A$.

4) $\left|\log _{2}\right| D \mathcal{G}_{B(k)}(x) \frac{h\left(\mathcal{G}_{B(k)}(x)\right)}{h(x)}\left|+\left(N_{k}-M\right)\right| \leq 2 \rho k$ for $1 \leq k$ and $x \in A$.

Proof. — 1) and 2) follow from Lemma 5.3 using estimates from Lemma 5.2.

3) Since $\left|D P_{a}\right| \leq 4$ on $[-\beta, \beta] \subseteq[-2,2]$ and $\mathcal{G}_{J}$ sends $A$ into $J$ with bounded distortion.

4) Rewrite 3) as $\left|\log _{2}\right| D \mathcal{G}_{J(\ell)}(x) h\left(\mathcal{G}_{J(\ell)}(x)\right) / h(x)\left|+n_{\ell}\right| \leq n_{\ell}+c$. Use the chain rule with 2 ) for $n_{\ell} \leq \bar{M}$ and 3 ) else wise. For strongly regular parameters $4^{\bar{M}-M} \ll \rho$ and

$$
\sum_{\substack{\ell=1 \\ n_{\ell} \geq \bar{M}}}^{k-1}\left(n_{\ell}+c\right)<\rho k+c \sum_{\substack{\ell=1 \\ n_{\ell} \geq \bar{M}}}^{k-1} \frac{n_{\ell}}{\bar{M}}<\left(1+c \bar{M}^{-1}\right) \rho k .
$$

Thus $\left(1+c \bar{M}^{-1}\right) \rho k+c k 4^{\bar{M}-M}<2 \rho k$.

TOME $131-2003-\mathrm{N}^{\mathrm{O}} 3$ 
Proof of Proposition 5.1. - 1) Set

$$
y:=\widetilde{\mathcal{G}}_{k}(x):=P_{a} \circ \mathcal{G}_{k}^{ \pm}(x)=P_{a}\left(Q^{-1} \circ \mathcal{G}_{B(k)}\right)(x) .
$$

By Proposition 4.2, for strongly regular parameters $\mathcal{G}_{B(k)}$ is a composition of $\mathcal{G}_{C_{n}^{ \pm}}$with $2 \leq n<\bar{M}$. Also $c^{-1} h(x) \leq h\left(\mathcal{G}_{B(k)}(x)\right) \leq \operatorname{ch}(x)$. As $k \leq q \ll$ $4^{M-\bar{M}}$, combining 1) and 2) of Lemma 5.4 with Lemma 5.2 yields

$$
c^{-1} 2^{-M-N_{k}} \leq\left|D \widetilde{\mathcal{G}}_{k}(x)\right| \leq c 2^{-M-N_{k}}
$$

$y=P_{a}\left((y-a)^{\frac{1}{2}}\right)$ implies $\left|D\left(P_{a}^{-1}\right)^{ \pm}(y)\right|=\frac{1}{2}|y-a|^{-\frac{1}{2}}$. Bounded distortion for $\widetilde{\mathcal{G}}_{k}$ yields

$$
c^{-1}\left|D \widetilde{\mathcal{G}}_{k}(x)\right| \leq \frac{|y-a|}{\left|x-P_{a}^{N_{k}-1}(0)\right|} \leq c\left|D \widetilde{\mathcal{G}}_{k}(x)\right| .
$$

For $x \in D_{k}$ and $P^{N_{k}}(0) \in J(k)$ with $n_{k}<\bar{M}$, Lemma 5.2 yields

$$
c^{-1} 2^{-n_{k}} \leq\left|x-P_{a}^{N_{k}}(0)\right| \leq c 2^{-n_{k}} .
$$

The statement now follows from the chain rule. For $k=0$ note that $c^{-1} \leq$ $\left|x-P_{a}^{M-1}(0)\right| \leq c$.

2) Lemma 5.4 part 4) yields $c^{-1} 2^{-M-N_{k}-2 \rho k} \leq\left|D \widetilde{\mathcal{G}}_{k}(x)\right| \leq c 2^{-M-N_{k}+2 \rho k}$. As above the chain rule and the bounded distortion argument yield

$$
c^{-1} 2^{-\frac{1}{2}\left(M+N_{k}\right)-\rho k} \leq\left|D \mathcal{G}_{k}^{ \pm}(x)\right| \cdot\left|x-P_{a}^{N_{k}}(0)\right|^{\frac{1}{2}} \leq c 2^{-\frac{1}{2}\left(M+N_{k}\right)+\rho k} .
$$

$J(k)$ is regular, so by Proposition 2.2 it is adjacent to a regular interval $J^{\prime} \subseteq \widehat{J}$ of order $n_{k}+3$ contained in the image of $Q$. Bounded distortion of $\mathcal{G}_{J(k)}$ and part 3) of Lemma 5.4 imply $c^{-1} 4^{-n_{k}} \leq c^{-1}|J(k)| \leq\left|J^{\prime}\right| \leq\left|x-P^{N_{k}}(0)\right| \leq c$ for $x \in D_{k}$ and the result follows.

3) $\widetilde{\mathcal{G}}_{k, I}:=P_{a} \circ \mathcal{G}_{k, I}^{ \pm}$. By Proposition 4.2 and Proposition $2.6 \operatorname{ord}(I)=n_{k}-1$. Proceed as in 1), as estimates of Lemma 5.42 ) also hold for $C_{1}^{ \pm}$. For bounded distortion of $\widetilde{\mathcal{G}}_{k, I}$ note that $c^{-1} \leq\left|x-P^{N_{k+1}-1}(0)\right| \leq c$ as $P^{N_{k+1}-1}(0) \notin A$ and $x \in D_{0}$.

4) By Proposition 2.6 and Proposition $4.2 \operatorname{ord}(I)<n_{k}<\rho k$. The same argument as in Lemma 5.4 part 4 ) holds for $\mathcal{G}_{B(k)} \circ \mathcal{G}_{I}$ (substituting $2 \rho k$ by $3 \rho k$ ). $c^{-1} 2^{-\left(M+N_{k}+\text { ord } I\right)-3 \rho k} \leq\left|D \widetilde{\mathcal{G}}_{k, I}(x)\right| \leq c 2^{-\left(M+N_{k}+\text { ord } I\right)+3 \rho k}$. Bounded distortion yields:

$$
\begin{aligned}
c^{-1} 2^{-\frac{1}{2}\left(M+N_{k}+\text { ord } I\right)-2 \rho k} \leq\left|D \mathcal{G}_{k, I}^{ \pm}(x)\right| \cdot \mid x- & \left.P^{N_{k}+\operatorname{ord}(I)}(0)\right|^{\frac{1}{2}} \\
& \leq c 2^{-\frac{1}{2}\left(M+N_{k}+\operatorname{ord} I\right)+2 \rho k} .
\end{aligned}
$$

One has $J(k) \subseteq \widehat{I} \backslash \operatorname{int}(I)$ by Proposition 2.6 so $c^{-1} \leq\left|x-P^{N_{k}+o r d(I)}(0)\right| \leq c$ as $x \in D_{0}$.

BULletin DE LA SOCIÉtÉ MATHÉMATIQUE DE FRANCE 
COROLlARY 5.5. - Strongly regular parameters satisfy the Collet-Eckmann condition:

$$
\liminf _{n \rightarrow \infty} \frac{1}{n} \ln \left|D P_{a}^{n}(a)\right|>0
$$

Proof. - Let $n, k \in \mathbb{N}$ with $N_{k} \leq n<N_{k+1}$ and $q<k$. For strongly regular parameters, the same reasoning as in Proposition 5.1 with $y=P_{a}^{N_{k}-1}(a) \in A$ yields

$$
c^{-1} 2^{M+N_{k}} \leq\left|D P_{a}^{N_{k}-1}(a)\right|=\left|D \widetilde{\mathcal{G}}_{k}\right|^{-1} \leq c 2^{M+N_{k}}
$$

for all $k \in \mathbb{N}$. If we had $\left|D P_{a}^{N_{k}-1}(a)\right| \leq c 2^{M+\frac{1}{2} N_{k}-n_{k}}$ for some $0 \leq i \leq n_{k}$ the chain rule would imply

$$
c^{-1} 2^{M+N_{k}+n_{k}} \leq\left|D P_{a}^{N_{k+1}-1}(a)\right| \leq c 2^{M+\frac{1}{2} N_{k}+n_{k}-2 i}
$$

leading to the contradiction $N_{k} \leq-2 i$. Therefore,

$$
\liminf _{n \rightarrow \infty} \frac{1}{n} \ln \left|D P_{a}^{n}(a)\right| \geq \liminf _{k \rightarrow \infty} \min _{0 \leq i \leq n_{k}} \frac{1}{k} \ln c 2^{M+\frac{1}{2} N_{k}-n_{k}} \geq c(1-\rho)>0
$$

as $2 k \leq N_{k} \leq n \leq N_{k+1}<(\bar{M}+\rho) k$.

\section{The Hausdorff dimension}

Consider an at most countable family of contractions $\mathcal{G}_{k}: D_{k} \rightarrow F$ where each $D_{k}$ is a closed subset of a compact invariant set

$$
F=\longdiv { \bigcup \mathcal { G } _ { k } ( D _ { k } ) }
$$

invariant with respect to all $\mathcal{G}_{k}$, where the closure is taken with respect to the Hausdorff topology. Let

$$
0<b_{k} \leq \frac{\left|\mathcal{G}_{k}(x)-\mathcal{G}_{k}(y)\right|}{|x-y|} \leq c_{k}<1
$$

for all $x \neq y \in D_{k}$. Assume $\sum c_{k} \leq 1$ and let $s_{+}, s_{-} \in \mathbb{R}$ be such that $\sum c_{k}^{s_{+}}=1=\sum b_{k}^{s_{-}}$.

Lemma 6.1. - Assume $\operatorname{dim}_{H}(\partial F) \leq s_{+}$and $\mathcal{G}_{i}\left(D_{i}\right) \cap \mathcal{G}_{j}\left(D_{j}\right)=\varnothing$. Then

$$
s_{-} \leq \operatorname{dim}_{H}(F) \leq s_{+}
$$

Proof. — In the finite case, see [7]. For the upper bound of the infinite case, consider $s>s_{+}$. By definition, for every $\delta>0$ and $\eta>0$ there is a $\delta$-cover $\left\{U_{i}\right\}$ of $F$ with $\sum\left(\operatorname{diam} U_{i}\right)^{s} \leq \mathcal{H}_{\delta}^{s}+\eta$. Then

$$
\left\{U_{i, k}\right\}_{i \in \mathbb{N}}:=\left\{\mathcal{G}_{k}\left(U_{i} \cap F\right)\right\}_{i \in \mathbb{N}}
$$

TOME $131-2003-\mathrm{N}^{\mathrm{O}} 3$ 
is a $c_{k} \delta$-cover of $\mathcal{G}_{k}(F)$ for each $k \in \mathbb{N}$. As $\operatorname{dim}_{H}\left(F_{\infty}\right) \leq s_{+}<s$, there is a $\delta$-cover $\left\{V_{j}\right\}$ of $\partial F$ such that $\left\{U_{i, k}, V_{j}\right\}$ is a $\delta$-cover of $F$ and

$$
\mathcal{H}_{\delta}^{s} \leq \sum_{i, k}\left(\operatorname{diam} U_{i, k}\right)^{s}+\sum_{j}\left(\operatorname{diam} V_{j}\right)^{s} \leq c\left(\mathcal{H}_{\delta}^{s}+\eta\right)+\eta
$$

for $c=\sum_{k} c_{k}^{s}<1$. As $\eta>0$ and $\delta>0$ were chosen arbitrary we get $\operatorname{dim}_{H}(F) \leq s$ for every $s>s_{+}$, hence $\operatorname{dim}_{H}(F) \leq s_{+}$. The lower bound follows from the finite case, as $F$ is also invariant for any finite subset of the infinite family of contractions. For details see [15].

TheOREM 6.2. - For strongly regular parameters there is a constant $c>0$ depending only on $M$ such that

$$
\operatorname{dim}_{H}(\mathcal{E})=\frac{\log _{2} M}{M}\left(1+\mathcal{O}\left(\frac{\log _{2} \log _{2} M}{M}\right)\right) .
$$

Proof. - By Definition 2.4, $\operatorname{dim}_{H}(\mathcal{E})=\operatorname{dim}_{H}(\widetilde{\mathcal{E}})$. For the upper bound, $\mathcal{K}_{k}$ and $\Gamma$ are countable by Proposition 2.6 so Corollary 4.3 insures we can apply Lemma 6.1 to $\widetilde{\mathcal{E}}$. Write

$$
\begin{aligned}
2 x_{k} & =N_{k}-n_{k}-c & & \text { for } k \leq q, \\
2 x_{k} & =N_{k}-2 n_{k}-2 \rho k-c & & \text { for } k>q, \\
2 x_{k, I} & =N_{k}+\operatorname{ord}(I)-4 \rho k-c & & \text { for } k>q \text { and } I \in \mathcal{K}_{k}
\end{aligned}
$$

and

$$
H\left(s, x_{0}, \ldots\right):=1-2^{-\frac{1}{2} s M+1}\left(\sum_{k=0}^{q} 2^{-s x_{k}}+\sum_{k>q} 2^{-s x_{k}}+\sum_{\substack{k>q \\ I \in \mathcal{K}_{k}}} 2^{-s x_{k}, I}\right) .
$$

Inserting the upper bounds of Proposition 5.1 yields $1-\sum_{\mathcal{G} \in \Gamma} c_{\mathcal{G}}^{s} \geq H\left(s, x_{0}, \ldots\right)$. As $\partial H / \partial s>0$ and $H\left(0, x_{0}, \cdots\right)<0$ values $s>0$ with $H\left(s, x_{0}, \ldots\right) \geq 0$ give upper bounds on $s_{+}$. The values of $x_{k}$ are larger than $2 x_{k} \geq M-\bar{M}+2 k-c$ for $k \leq q, 2 x_{k} \geq M+2(1-2 \rho) k-c$ for $k>q$ as $n_{k}<\rho k$ and $2 x_{k, I} \geq M+2(1-2 \rho) k-c$ for $k>q$ and $I \in \mathcal{K}_{k}$ as $\operatorname{ord}(I) \geq 1$. For strongly regular parameters $\operatorname{Card}\left(\mathcal{K}_{k}\right)<n_{k}<\rho k$ for $k>q$ by Proposition 2.6 and Proposition 4.2. Then, as $s>0$ and $(1+\rho k) 2^{-(1-2 \rho) s k}<2^{-c s k}$ we have

(2) $H\left(s, x_{0}, \ldots\right)$

$$
\quad \geq 1-2^{\left(\frac{1}{2}(c+\bar{M})-M\right) s+1}\left(\frac{1-2^{-(q+1) s}}{1-2^{-s}}+\frac{2^{-\frac{1}{2}(\bar{M}+c q) s}}{1-2^{-c s}}\right):=h\left(s, x_{0}, \ldots\right)
$$

Again $\partial h / \partial s>0$ and the Implicit Function Theorem insures the existence of a $C^{1}$ solution $s^{\infty}=s^{\infty}\left(x_{0}, x_{1}, \ldots\right)$ of $h\left(s, x_{0}, \ldots\right)=0$ with $s^{\infty} \geq s_{+}$. For our choice of constants $\rho$ and $\bar{M}$ we have $q \gg M$, and as $s>0$ there is some constant $c>0$

$$
c^{-1} s^{-1} \leq c^{-1}\left(1-2^{-c s}\right)^{-1} \leq c \frac{1-2^{-c s(q+1)}}{1-2^{-c s}} \leq c\left(1-2^{-c s}\right)^{-1} \leq c s^{-1} .
$$

BULletin DE LA SOCIÉtÉ MATHÉMATIQUE DE FRANCE 
As $h\left(\frac{2}{3} \log _{2} M / M, x_{0}, \ldots\right)<0$ we have $s^{\infty} \geq \frac{2}{3} \log _{2} M / M$. Set $s=\widehat{s} \log _{2} M / M$. With (3) we have

$$
h\left(s^{\infty}, x_{0}, \ldots\right)=0 \geq \frac{1}{2} M^{\left(1-\frac{\bar{M}+c}{M}\right) \widehat{s}}-\frac{c M}{\log _{2} M} .
$$

Therefore

$$
s_{\text {max }}:=\left(1-\frac{\bar{M}+c}{M}\right)^{-1}\left(1-\frac{\log _{2}\left(\log _{2} M / c\right)}{\log _{2} M}\right) \leq 1+\mathcal{O}\left(\frac{\log _{2} \log _{2} M}{\log _{2} M}\right)
$$

and $\operatorname{dim}_{H}(\mathcal{E}) \leq s_{+} \leq\left(\log _{2} M / M\right)\left(1+\mathcal{O}\left(\log _{2} \log _{2} M / \log _{2} M\right)\right)$

For the lower bound we apply the finite case of Lemma 6.1 to the attractor defined in Corollary 4.3 restricting to $k \leq q$. The computations are similar as above. Replace the upper bounds of Proposition 5.1 by the lower bounds and take upper bounds for the $x_{k}$ and $x_{k, I}$.

The following leads to a notable simplifications in Yoccoz's proof of Jakobson's Theorem:

Proposition 6.3. - For a strongly regular parameter and $M$ large enough

$$
\operatorname{Leb}\left(A \backslash \bar{W}_{n}\right) \leq c 2^{-\frac{1}{3} n} .
$$

Proof. - Recall $\bar{\ell} \in \mathbb{N}$ with $N_{\bar{\ell}}^{\prime} \leq n<N_{\bar{\ell}+1}^{\prime}$ (where $N_{\ell}^{\prime}=N_{\ell}+1$ or $N_{\ell}+3$ ). We have $A \backslash \bar{W}_{n} \subseteq \bigcup_{0 \leq k \leq \bar{\ell}} \varepsilon(n, k) \cup \varepsilon_{c}(n)$. With $\mathcal{G} \in \Gamma$ the inverse branch of an iterate $P_{a}^{m_{\mathcal{G}}}$ with $m_{\mathcal{G}} \in \mathbb{N}$ Proposition 3.1 yields

$$
\operatorname{Leb}\left(A \backslash \bar{W}_{n}\right):=\mu_{n} \leq\left|\widehat{A}_{\bar{\ell}}\right|+\sum_{\mathcal{G} \in \Gamma} \sup |D \mathcal{G}| \mu_{n-m_{\mathcal{G}}}
$$

Proposition 5.1 without the last distortion step yields

$$
\left|\widehat{A}_{\bar{\ell}}\right| \leq c 2^{-\frac{1}{2}\left(M+N_{\bar{\ell}}\right)+\rho \bar{\ell}} \leq c 2^{-\frac{1}{2}(M+(1-3 \rho) n)}
$$

for $q=\left[\rho^{-1} \bar{M}\right]<\bar{\ell}<n<N_{\bar{\ell}}+\rho \bar{\ell}+3$ and $\sup |D \mathcal{G}| \leq c 2^{-\frac{1}{2}\left(M+m_{\mathcal{G}}\right)+\rho m_{\mathcal{G}}}$. With $\widehat{\mu}_{n}=2^{\frac{1}{3} n} \mu_{n}$ as all $m_{\mathcal{G}} \geq M-1$ are distinct, we have

$$
\begin{aligned}
c^{-1} 2^{\frac{1}{2} M} \widehat{\mu}_{n} & \leq 2^{-\left(\frac{1}{6}-2 \rho\right) n}+\sum_{\mathcal{G} \in \Gamma} 2^{-\left(\frac{1}{6}-\rho\right) m_{\mathcal{G}}} \widehat{\mu}_{n-m_{\mathcal{G}}} \\
& \leq c+\sup _{n^{\prime} \leq n-m_{\mathcal{G}}} \widehat{\mu}_{n^{\prime}} \sum_{m \geq m_{\mathcal{G}}} 2^{-\frac{1}{12} m} \leq c
\end{aligned}
$$

for $\rho<\frac{1}{12}$.

TheOREM 6.4. - For a set of parameters $\mathcal{R}$ with

$$
\lim _{\delta \rightarrow 0} \frac{\operatorname{Leb}(\mathcal{R} \cap[-2,-2+\delta))}{\delta}=1
$$

TOME $131-2003-\mathrm{N}^{\mathrm{O}} 3$ 
there exists an absolutely continuous invariant probability measure for $P_{a}$ with $a \in \mathcal{R}$ and the Hausdorff dimension of the $\epsilon$-weakly expanding set $\mathcal{E}$ for $c 2^{-M}<\epsilon \leq|\alpha|$ with a given constant $c>0$ is

$$
\operatorname{dim}_{H}(\mathcal{E})=\frac{\log _{2} M}{M}\left(1+\mathcal{O}\left(\frac{\log _{2} \log _{2} M}{\log _{2} M}\right)\right)
$$

where constants depend only on $M$.

Proof. - The partition $W$ satisfies the hypothesis of the Folklore Theorem (see [12]) by construction and by Proposition 6.3 (for the full measure and summability), implying the existence of an a.c.i.p. for strongly regular parameters. The Hausdorff dimension estimate for the $|\alpha|$-weakly expanding set is proved in Theorem 6.2. For $\epsilon \leq|\alpha|$ the $\epsilon$-weakly expanding set is contained in the $\alpha$-weakly expanding set, so the upper bound holds. For the lower bound and $\left|\alpha^{M-2}\right|<\epsilon$, the $\epsilon$-weakly expanding set is generated by the contractions $\mathcal{G} \in \Gamma$ of Definition 3.3 and therefore this set must be the same as the $|\alpha|$-weakly expanding set. Finally we must prove that the strongly regular parameters have a Lebesgue density point at -2 . This is proved using the following standard large deviation argument (see [17] or [15] for details). Applying Lemmas 5.2 and 5.4 and using the particular form of the quadratic map one obtains for $a \in\left(a_{M-1}, a_{M-2}\right)$ that

$$
\left|\frac{\mathrm{d}}{\mathrm{d} a} P_{a}^{-n}(x)-\frac{1}{3}\right| \leq c n 4^{-n}
$$

for $x \in\left[\alpha^{n}, \alpha^{n-1}\right]$ and $n>0$, as well as

$$
\left|\frac{\mathrm{d}}{\mathrm{d} a} \mathcal{G}_{C_{n}^{ \pm}}(x)\right| \leq c 2^{n}
$$

for $x \in A$ and $2 \leq n \leq M-2$ and

$$
\left|\frac{\mathrm{d}}{\mathrm{d} a} \mathcal{G}_{J}(x)\right| \leq c 2^{4 \operatorname{ord}(J)}
$$

for $x \in A$ and $J$ regular. Write $\widetilde{\Delta}_{n}:=\left\{a<0 ; P_{a}^{n}(0)= \pm \alpha(a)\right\}$ and

$$
U(k)=U(k)(a)
$$

for the connected component in $\mathbb{R} \backslash \widetilde{\Delta}_{N_{k}}$ of a strongly regular parameter $a$. Observe that regular intervals of order $<N_{k}$ vary continuously on each $U(k)$ and the sequence $M=N_{1}<N_{2}<\cdots<N_{k}$ is constant on each $U(k)$. Let

$$
N(k)= \begin{cases}\bar{M}-1 & \text { if } k \leq q=\left[\rho^{-1} \bar{M}\right], \\ {[\rho k]} & \text { if } k>q,\end{cases}
$$

and write $\left[\gamma_{i}^{-}(a), \gamma_{i}^{+}(a)\right]$ with $0 \leq i \leq r$ for the maximal regular intervals of order smaller than $m<N(k)$. This partition of $A$ induces a partition 
$a^{-}=a_{0}^{-}<a_{0}^{+} \leq a_{1}^{-}<a_{1}^{+} \leq \cdots \leq a_{r}^{-}<a_{r}^{+}=a^{+}$of $U(k)=\left(a^{-}, a^{+}\right)$and the above estimates imply

$$
\left|\frac{\mathrm{d}}{\mathrm{d} a} P_{a}^{-(M-1)} \circ \mathcal{G}_{B(k)}\left(\gamma_{i}^{ \pm}(a)\right)-\frac{1}{3}\right| \leq c 2^{-M},
$$

hence $a_{i+1}^{-}-a_{i}^{+} \leq c\left(a^{+}-a^{-}\right) \max _{a \in U(k)}\left(\gamma_{i+1}^{-}(a)-\gamma_{i}^{+}(a)\right)$. Proposition 6.3 implies $\sum_{0 \leq i \leq r} \max _{a \in U(k)}\left(\gamma_{i+1}^{-}(a)-\gamma_{i}^{+}(a)\right) \leq c 2^{-\frac{1}{3} m}$, and therefore

$$
\sum_{0 \leq i \leq r} a_{i+1}^{-}-a_{i}^{+} \leq c\left(a^{+}-a^{-}\right) 2^{-\frac{1}{3} m} .
$$

Write $U_{k}=\bigcup_{a} U(k)(a)$ where the union is taken over all strongly regular parameters $a$ with $P_{a}(0) \in\left(\alpha^{M-1}, \alpha^{M-2}\right)$. Then define the random variable

$$
X_{k}=X_{k}(a)= \begin{cases}0 & \text { if } a \in U_{k-1} \backslash U_{k}, \\ \operatorname{ord}(J) & \text { if } T^{k}(0) \in \operatorname{int} J, J \in \mathcal{J} \text { with } \operatorname{ord}(J) \leq N(k), \\ N(k)+1 & \text { elsewise. }\end{cases}
$$

The above estimates yield

$$
\operatorname{Leb}\left(X_{k}>m \mid X_{1}, \ldots, X_{k-1}\right) \leq c 2^{-\frac{1}{3} m}
$$

and as $S_{k}(a):=\sum_{\substack{1 \leq i \leq k \\ X_{i} \geq \bar{M}}} X_{i}$ is constant on each $U(k)$ we have that

$$
\int_{a_{M-1}}^{a_{M-2}} 2^{\theta S_{k}(a)} \mathrm{d} a \leq\left(1+c 2^{\left(\theta-\frac{1}{3}\right) \bar{M}}\right)^{k}\left(a_{M-2}-a_{M-1}\right) .
$$

Chebyshev's inequality for the non-negative random variable $X=2^{\theta S_{k}}-1$ implies that

$$
\frac{\operatorname{Leb}\left(a \in\left(a_{M-1}, a_{M-2}\right) \mid S_{k}(a) \geq \rho k\right)}{\left(a_{M-2}-a_{M-1}\right)} \leq \frac{\left(1+c 2^{\left(\theta-\frac{1}{3}\right) \bar{M}}\right)^{k}-1}{2^{\theta \rho k}-1}=: u_{k} .
$$

For any $\theta<\frac{1}{3}$ and for $k \leq \rho^{-1}$ we have $u_{k} \leq c \rho^{-1} 2^{\left(\theta-\frac{1}{3}\right) \bar{M}}$. Write $j=[\rho k]$. Then for $\rho^{-1}<k \leq 2^{-\left(\theta-\frac{1}{3}\right) \bar{M}}$ and any $\theta<\frac{1}{3}$ we have $u_{k} \leq c \rho^{-1} j 2^{\left(\theta-\frac{1}{3}\right) \bar{M}-\theta j}$. For $k>2^{\left(\frac{1}{3}-\theta\right) \bar{M}}$ we have $u_{k} \leq c \lambda^{k}$ where $\lambda=\left(1+c 2^{\left(\theta-\frac{1}{3}\right) \bar{M}}\right) 2^{-\theta \rho} \leq\left(1-c^{-1} \rho\right)$ (recall that $\bar{M} 2^{-\bar{M}} \ll \rho$, so choose $2^{-\delta \bar{M}} \leq \rho$ with $\delta<\frac{1}{3}-\theta$ for instance). Observe that $c$ may depend on $\theta$, but not on $\bar{M}$ or $M$, provided these are large enough. Splitting the sum and using these bounds,

$$
\sum u_{k} \leq c \rho^{-2} 2^{\left(\theta-\frac{1}{3}\right) \bar{M}}+\left(c \rho^{-1} 2^{\left(\theta-\frac{1}{3} \bar{M}\right.}\right) \sum_{j \geq 1} j 2^{-\theta j}+c \rho^{-1} 2^{-c^{-1} \rho 2^{-\left(\theta-\frac{1}{3}\right) \bar{M}}} .
$$

Finally, for $0<\widehat{\theta}<\frac{1}{3}-\theta$ we get for $M$ large enough $\sum_{k \geq 1} u_{k} \leq 2^{-\widehat{\theta} \bar{M}}$. Taking the limit as $M$ goes to infinity completes the proof. 


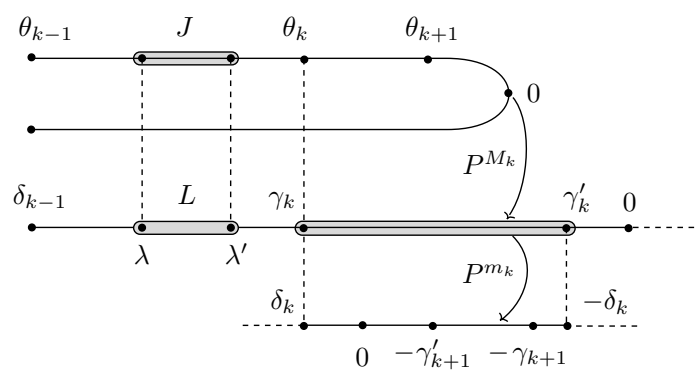

Figure 2.

\section{Generalization to arbitrary epsilon}

The expansion required in the Folklore Theorem to build an absolutely continuous invariant measure is arbitrary. So the following definition is natural:

Definition 7.1. - Let $B(0, \epsilon)$ denote the ball of radius $\epsilon$ centered at 0 . A point $x$ belongs to the primary $\epsilon$-weakly expanding set $\widetilde{\mathcal{E}}_{\epsilon}$ if no neighborhood $U$ of $x$ can be sent univalently by any $P_{a}^{n}$ for $n>0$ onto an interval containing $B(0, \epsilon)$. A point $x$ belongs to the $\epsilon$-weakly expanding set $\mathcal{E}_{\epsilon}$ if $P_{a}^{n}(x) \in \widetilde{\mathcal{E}}_{\epsilon}$ for some $n \geq 0$. Note that $\widetilde{\mathcal{E}}_{\epsilon}=\widetilde{\mathcal{E}}$ for $\left|\widetilde{\alpha}^{M-2}\right| \leq \epsilon$.

Definition 7.2. - Set $\Delta_{n}:=P^{-n}(\{\alpha,-\alpha\})$ for $n \geq 0$. Then $\gamma \in \Delta_{n} \backslash \Delta_{n-1}$ (or $\gamma \in \Delta_{0}$ for $n=0$ ) is said to be of order $\operatorname{ord} \gamma=n$. Define

$$
\begin{aligned}
& \mathcal{D}=\left\{\alpha \leq \gamma<0 ; \exists n \geq 0 \text { with } \gamma \in \Delta_{n} \text { and }(\gamma,-\gamma) \cap \Delta_{n}=\varnothing\right\}, \\
& \widetilde{\mathcal{D}}=\left\{\alpha \leq \gamma<0 ; \exists n \geq 0 \text { with } \gamma \in \Delta_{n} \text { and }(\gamma,-\gamma) \cap \Delta_{n-1}=\varnothing\right\} .
\end{aligned}
$$

Then $\widetilde{\alpha}^{n} \in \mathcal{D} \subseteq \widetilde{\mathcal{D}}$ and for $\gamma, \gamma^{\prime} \in \widetilde{\mathcal{D}}, \gamma<\gamma^{\prime}$ implies ord $\gamma \leq$ ord $\gamma^{\prime}$. If $\gamma \in \mathcal{D}$ the inequality is strict. An interval $\left[\gamma, \gamma^{\prime}\right]$ is lateral if $\gamma<\gamma^{\prime}$ are consecutive in $\widetilde{\mathcal{D}}$. An interval $[\gamma,-\gamma]$ with $\gamma \in \mathcal{D}$ is central. For a lateral interval $J$, the order $\operatorname{ord}(J)$ is the largest integer $n$ for which $\left.P^{n}\right|_{J}$ is monotone.

Lemma 7.3. - Let $J=\left[\gamma, \gamma^{\prime}\right]$ be lateral of order $n$. Then $P^{n}(J):=[\delta,-\delta]$ is central. The complexity $c(J):=\operatorname{ord}(\delta)$ is defined as the order of $\delta$. Then $\operatorname{ord}(J)+c(J)=\operatorname{ord} \gamma^{\prime}$ and $\delta=\alpha$ iff $\operatorname{ord} \gamma<\operatorname{ord} \gamma^{\prime}$.

Proof. - As $0 \in P^{n}(J)$ and as $\gamma, \gamma^{\prime} \in \widetilde{\mathcal{D}}$ are consecutive $\operatorname{int}\left(P^{n}(J)\right) \cap$ $\Delta_{\text {ord } \gamma^{\prime}-n}=\varnothing$ so $P^{n}(J)$ is central. If $P^{n}(J)=[\alpha,-\alpha]$ then ord $\gamma<$ ord $\gamma^{\prime}=n$. Otherwise $P^{n+1}(\gamma)=P^{n+1}\left(\gamma^{\prime}\right)$ so $n<$ ord $\gamma^{\prime}=$ ord $\gamma$.

Now assume $P_{a}^{n}(0) \neq \alpha$ and $P_{a}$ non renormalizable.

BULLETIN DE LA SOCIÉTÉ MATHÉMATIQUE DE FRANCE 
Definition 7.4. - Set $M_{1}=M, \theta_{0}=\widetilde{\alpha}^{M-1}, \delta_{0}=\alpha$. Define inductively $M_{k+1}=M_{k}+m_{k}$ where $m_{k}$ is the order of the lateral interval $\left[\gamma_{k}, \gamma_{k}^{\prime}\right]$ containing $\pm P^{M_{k}}(0)$ (see Fig. 2). Let $\left[\delta_{k},-\delta_{k}\right]:=P^{m_{k}}\left(\left[\gamma_{k}, \gamma_{k}^{\prime}\right]\right)$. Then

$$
\left[\gamma_{k+1}, \gamma_{k+1}^{\prime}\right] \subseteq\left[\delta_{k},-\delta_{k}\right] .
$$

Let $\left[\theta_{k},-\theta_{k}\right]$ be the connected component of $P^{-M_{k}}\left( \pm\left[\gamma_{k}, \gamma_{k}^{\prime}\right]\right)$ containing the critical point. For strongly regular parameters, we have $\left(N_{\ell}\right)_{\ell \geq 1}=\left(M_{s(\ell)}\right)_{\ell \geq 1}$ with $s(\ell+1)=s(\ell)+r$ for some $r>0$.

Proposition 7.5. - Let $\ell \in \mathbb{N}$ satisfy $s(\ell) \leq k<s(\ell+1)$. Then $\left[\theta_{k},-\theta_{k}\right]$ is a central interval. A lateral interval $J=\left[\gamma, \gamma^{\prime}\right] \subseteq\left[\theta_{k-1}, \theta_{k}\right]$ of order $\operatorname{ord}(J)=n$ is the pre-image by $P_{a}^{-M_{k}}$ of one of either

- a central interval $K \subseteq\left[\gamma_{k}^{\prime},-\gamma_{k}^{\prime}\right]$ if $n=M_{k}$. Then $c(J)=\operatorname{ord}\left(\gamma_{k}^{\prime}\right)$.

- a lateral interval $L$ (or its symmetric $-L)$ if $n>M_{k}$. Then $n=\operatorname{ord}(L)+$ $M_{k}$ and $c(J)<\operatorname{ord}\left(\gamma_{k}^{\prime}\right)$.

For strongly regular parameters $\operatorname{ord}\left(\gamma_{k}^{\prime}\right)<\bar{M}$ for $\ell \leq q=\left[\rho^{-1} \bar{M}\right]$ and $\operatorname{ord}\left(\gamma_{k}^{\prime}\right)<\rho \ell \leq \rho k$ elsewise.

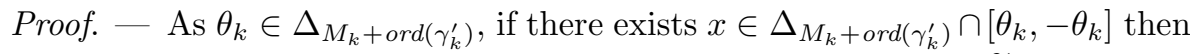
$\pm P^{M_{k}}(x) \in \Delta_{\operatorname{ord}\left(\gamma_{k}^{\prime}\right)} \cap\left[\gamma_{k}, \gamma_{k}^{\prime}\right]$ and $\gamma_{k}, \gamma_{k}^{\prime}$ are not consecutive in $\widetilde{\mathcal{D}}$. If $n<M_{k}$ then $0 \in P^{n}(J) \subseteq P^{n}\left(\left[\theta_{k-1}, \theta_{k}\right]\right)$ and for some $0 \leq i \leq n-1$ the interval $\left[\gamma_{i}, \gamma_{i}^{\prime}\right]$ would have order less than $m_{i}$. If $n=M_{k}$ then $0 \in P^{n}(J) \subseteq P^{n}\left(\left[\theta_{k-1}, \theta_{k}\right]\right)$ hence $\pm P^{n}\left(\theta_{k}\right)=\gamma_{k}^{\prime}$ and $P^{n}(J) \subseteq\left[\gamma_{k}^{\prime},-\gamma_{k}^{\prime}\right]$. Then

$$
c(J)=\operatorname{ord}\left(P^{n}(\gamma)\right)=\operatorname{ord}\left(P^{n}\left(\gamma^{\prime}\right)\right) \geq \operatorname{ord}\left(\gamma_{k}^{\prime}\right)
$$

by Lemma 7.3 and since $\alpha \leq \gamma_{k}<\gamma_{k}^{\prime} \in \widetilde{\mathcal{D}}$. But if $\operatorname{ord}\left(P^{n}(\gamma)\right)>\operatorname{ord}\left(\gamma_{k}^{\prime}\right)$ then $\operatorname{ord}(\gamma)>\operatorname{ord}\left(\theta_{k}\right)$ contradicting $\gamma \in \widetilde{\mathcal{D}}$. If $M_{k}<n$ then $0 \notin P^{M_{k}}(J)$. Denote by $L:=\left[\lambda, \lambda^{\prime}\right]$ the negative component of $\pm P^{M_{k}}(J)$. Then either $\lambda^{\prime} \leq \gamma_{k}$ or $\gamma_{k}^{\prime} \leq \lambda$ or $\pm P_{a}^{M_{k}}(J)=\left[\gamma_{k}, \gamma_{k}^{\prime}\right]$. In the first case $\operatorname{ord}(\lambda) \leq \operatorname{ord}\left(\lambda^{\prime}\right) \leq \operatorname{ord}\left(\gamma_{k}\right)$, and assuming $\lambda \notin \widetilde{\mathcal{D}}$ implies the existence of some $x \in \Delta_{\text {ord }(\lambda)-1} \cap\left(\lambda, \gamma_{k}\right)$ since $\gamma_{k} \in$ $\widetilde{\mathcal{D}}$. Then $P_{a}^{-M_{k}}( \pm x) \in\left(\gamma, \theta_{k}\right)$ with $\operatorname{ord}\left(P_{a}^{-M_{k}}( \pm x)\right)<\operatorname{ord}(\gamma)$ contradicting $\gamma \in \widetilde{\mathcal{D}}$. This reasoning implies $\lambda, \lambda^{\prime} \in \widetilde{\mathcal{D}}$ and these points are consecutive since $\gamma$ and $\gamma$ are. If $\gamma_{k}^{\prime} \leq \lambda$ then $\operatorname{ord}\left(\gamma_{k}^{\prime}\right)=\operatorname{ord}\left(\lambda^{\prime}\right)=\operatorname{ord}(\lambda)$ since $\gamma_{k}^{\prime} \in \widetilde{\mathcal{D}}$ and $\operatorname{ord}(\gamma) \leq \operatorname{ord}\left(\gamma^{\prime}\right) \leq \operatorname{ord}\left(\theta_{k}\right)$. Assuming $\lambda \notin \widetilde{\mathcal{D}}$ (or $\left.\lambda^{\prime} \notin \widetilde{\mathcal{D}}\right)$ implies the existence of some $x \in \Delta_{\operatorname{ord}(\lambda)-1} \cap(\lambda, 0)$ which contradicts $\gamma_{k}^{\prime} \in \widetilde{\mathcal{D}}$. As above $\lambda$ and $\lambda^{\prime}$ are consecutive in $\widetilde{\mathcal{D}}$ as $\gamma$ and $\gamma^{\prime}$ are. If $\pm P_{a}^{M_{k}}(J)=\left[\gamma_{k}, \gamma_{k}^{\prime}\right]$, then $J$ is obviously the pre-image of a lateral interval. Finally by Lemma 7.3 we have $c(L)<c(L)+\operatorname{ord}(L)=\operatorname{ord}\left(\lambda^{\prime}\right) \leq \operatorname{ord}\left(\gamma_{k}^{\prime}\right)$ and

$$
\operatorname{ord}\left(\gamma_{k}^{\prime}\right) \leq \sum_{i=k}^{s(\ell+1)-1} m_{i} \leq \sum_{i=s(\ell)}^{s(\ell+1)-1} m_{i}=n_{\ell} .
$$

TOME $131-2003-\mathrm{N}^{\mathrm{O}} 3$ 
Proposition 4.2 completes the proof.

Now consider only strongly regular parameters $a$ with $P_{a}(0) \in\left(\widetilde{\alpha}^{M-1}, \widetilde{\alpha}^{M-2}\right)$.

Lemma 7.6. - The number $\nu(m, c)$ of lateral intervals of order $m$ and complexity c satisfies $\nu(m, 0) \leq 1$ and $\nu(m, c) \leq 2^{t}$ for $m \leq 2 \rho^{-(t+1) \bar{M}}$ if $c<\bar{M}$ or for $m \leq 2 \rho^{-(t+1)} c$ if $\bar{M} \leq c$.

Proof. - Assume $\nu(m, 0) \geq 2$. Then the right endpoint of the left one of two lateral intervals of order $m$ and complexity 0 would have higher order than the left endpoint of the right interval by Lemma 7.3 and thus wouldn't belong to $\widetilde{\mathcal{D}}$. This contradiction implies $\nu(m, 0) \leq 1$. For $m \leq M-1$ the only lateral interval of order $m$ is $\left[\widetilde{\alpha}^{m-1}, \widetilde{\alpha}^{m}\right]$ which has complexity zero thus $\nu(m, c)=0$. By Proposition 4.2 and Proposition 7.5

$$
\begin{array}{ll}
\nu(m, c) \leq 1 & \text { for } \quad M \leq m \leq 2 \rho^{-1} \bar{M}<N_{\left[\rho^{-1} \bar{M}\right]} \text { if } c<\bar{M}, \\
\nu(m, c)=0 & \text { for } \quad M \leq m \leq 2 \rho^{-1} c<N_{\rho^{-1} c} \quad \text { if } \bar{M} \leq c .
\end{array}
$$

For $2 \rho^{-1} \bar{M}<m$ let $j \in \mathbb{N}$ satisfy $\operatorname{ord}\left(\theta_{j-1}\right)<m+c \leq \operatorname{ord}\left(\theta_{j}\right)$. As $c>0$ both endpoints of a lateral interval contributing to $\nu(m, c)$ have order $m+c$ by Lemma 7.3. As $\theta_{j} \in \mathcal{D}$ such an interval is contained in $\left[\theta_{j-1}, \theta_{j}\right]$. By Proposition $7.5 \nu(m, c) \leq \max \left\{1,2 \nu\left(m-M_{j}, c\right)\right\}$ and for strongly regular parameters $m-M_{j} \leq$ ord $\gamma_{j}^{\prime}<\rho j \leq \rho M_{j} \leq \rho m$. Thus $\nu(m, c) \leq 2^{t}$ for $m \leq 2 \rho^{-(t+1)} \bar{M}$ if $c<\bar{M}$ or for $m \leq 2 \rho^{-(t+1)} c$ if $\bar{M} \leq c$.

Definition 7.7. - Set $u_{0}:=\widetilde{\alpha}^{M-2}$ and consider the nested sequence of central intervals $\left[u_{s},-u_{s}\right]:=(A(s))_{s \geq 0}$ with $A(s)$ from Definition 2.5 for $s \geq 1$. Define inductively the decomposition $\mathcal{P}([\gamma,-\gamma])$ of a central interval $[\gamma,-\gamma]$ as follows

- If $\gamma<u_{0}$ then $\mathcal{P}([\gamma,-\gamma])=\left\{C_{n}^{ \pm} \mid \operatorname{ord}(\gamma)<n \leq M-2\right\} \cup\{A(0)\}$.

- If $u_{s} \leq \gamma<u_{s+1}$ then, with $J \subseteq\left[\gamma, u_{s+1}\right]$ lateral and $I$ an element of the decomposition $\mathcal{P}\left(P_{a}^{\text {ord }(J)}(J)\right)$

$$
\mathcal{P}([\gamma,-\gamma])=\{ \pm J ; c(J)=0\} \cup\left\{P^{-\operatorname{ord}(J)}(I) \subseteq J ; c(J)>0\right\} \cup\{A(s+1)\} .
$$

Call an element of the decomposition of $A(s)$ maximal if it is a pre-image of some $A(r)$ and is not contained in any pre-image of $A(t)$ with $t<r$ (or of $A$ if $r=1)$. Let $\widehat{\nu}(s, r)$ be the number of maximal pre-images of $A\left(r^{\prime}\right), r^{\prime} \geq r$ in the decomposition of $A(s)$.

Lemma 7.8. - Let $\omega:=\rho \bar{M} \ll 1$. For $s \geq 1$ we have $\widehat{\nu}(s, r)=0$ if $s<r-1$, $\widehat{\nu}(s, r)=1$ if $r-1 \leq s<\omega^{-1} r$ and

$$
\widehat{\nu}(s, r) \leq\left(c \bar{M} \omega s^{2}\right)^{2 \log _{2}(r / s) / \log _{2} \omega} \text { elsewise }
$$

BULletin DE LA SOCIÉtÉ MATHÉMATIQUE DE FRANCE 
Proof. - $A(s+1)$ contributes to $\widehat{\nu}(s, r)$ if and only if $s \geq r-1$. The decomposition of $P_{a}^{\operatorname{ord}(J)}(J)$ for a lateral interval $J \subset\left[u_{s}, u_{s+1}\right]$ of order $m$ and complexity $c$ contains maximal pre-images of $A\left(r^{\prime}\right), r^{\prime} \geq r$ if and only if $c \geq N_{r-1}$. For $\omega s \leq \ell \in \mathbb{N}$ and $c \geq N_{\ell}$ we have $m \leq 2 \rho^{-1} c$ since $2 s \leq N_{s+1}-M \leq(\bar{M}+\rho) s+\rho$. So by Lemma $7.6, \nu(m, c)=0$. Therefore we can write

$$
\widehat{\nu}(s, r) \leq 1+2 \sum_{\ell=r-1}^{\omega s-1} \sum_{\substack{N_{s}<m+c \leq N_{s+1} \\ N_{\ell} \leq c \leq N_{\ell+1}}} \nu(m, c) \widehat{\nu}(\ell, r) .
$$

For $\omega s<r$ the sum is empty and we obtain the first two inequalities. For $r \leq \omega s$ we have

$$
\widehat{\nu}(s, r) \leq 1+2 \bar{M}^{2} s \sum_{\ell=r-1}^{\omega s-1} \ell \widehat{\nu}(\ell, r) \max _{\substack{N_{s}<m+c \leq N_{s+1} \\ N_{\ell} \leq c \leq N_{\ell+1}}} \nu(m, c) .
$$

For $t=\left[\log _{2} s\right]-1$ we have $m \leq 2 \rho^{-(t+1)} c$ so

$$
\max _{\substack{N_{s} \leq m+c \leq N_{s+1} \\ N_{\ell} \leq c \leq N_{\ell+1}}} \nu(m, c) \leq 2^{t} \leq s
$$

As $\widehat{\nu}(\ell, r)$ increasing with $\ell$,

$$
\widehat{\nu}(s, r) \leq c \bar{M}^{2} s^{2} \sum_{\ell=r-1}^{\omega s-1} \ell \widehat{\nu}(\ell, r) \leq \prod_{i=0}^{k}\left(c \bar{M}^{2} s^{4} \omega^{2(2 i+1)}\right) \widehat{\nu}\left(\omega^{k} s, r\right) .
$$

Finally for $k=\left[\log _{2}(r / s) / \log _{2} \omega\right]$ we have $\omega^{k} s<\omega^{-1} r$ and $\widehat{\nu}\left(\omega^{k} s, r\right)=1$ hence $\widehat{\nu}(s, r) \leq\left(c \bar{M} \omega s^{2}\right)^{2} \log _{2}(r / s) / \log _{2} \omega$.

TheOREM 7.9. - For a set of parameters $\mathcal{R}$ with

$$
\lim _{\delta \rightarrow 0} \frac{\operatorname{Leb}(\mathcal{R} \cap[-2,-2+\delta))}{\delta}=1
$$

there exists an absolutely continuous invariant probability measure for $P_{a}$ with $a \in \mathcal{R}$ and the Hausdorff dimension of the $\epsilon$-weakly expanding set $\mathcal{E}_{\epsilon}$ for $c 2^{-M}<\epsilon \leq|\alpha|$ with a given constant $c>0$ is

$$
\operatorname{dim}_{H}(\mathcal{E})=\frac{\log _{2} M}{M}\left(1+\mathcal{O}\left(\frac{\log _{2} \log _{2} M}{\log _{2} M}\right)\right)
$$

with constants depending on $M$. For $0<\epsilon \leq|\alpha|$ arbitrary, we have

$$
\operatorname{dim}_{H}\left(\mathcal{E}_{\epsilon}\right)=\mathcal{O}\left(\frac{\log |\log \epsilon|}{|\log \epsilon|}\right) .
$$

Proof. - For the first part of the claim see Theorem 6.4. For the estimate of the dimension for arbitrary $\epsilon$, note that $\left[\widetilde{\alpha}^{M-2}, \widetilde{\alpha}^{M-1}\right]$ is lateral of complexity zero and for strongly regular parameters $\widetilde{\alpha}^{M-1}=u_{1}$ is the left endpoint of a lateral interval of complexity zero. So for $\epsilon \geq\left|\widetilde{\alpha}^{M-1}\right|$ we obtain $\operatorname{dim}_{H}\left(\widetilde{\mathcal{E}}_{\epsilon}\right)$

TOME $131-2003-\mathrm{N}^{\mathrm{O}} 3$ 
as in Theorem 6.2. For our parameters both components of $\widehat{A}(k) \backslash \operatorname{int}(A(k))$ are contained in lateral intervals of zero complexity. So for $\epsilon<\left|\widetilde{\alpha}^{M-1}\right|, \widetilde{\mathcal{E}}_{\epsilon}$ is contained in the maximal pre-images of some $A\left(r^{\prime}\right)$ with $r^{\prime} \geq r$, and $r \in \mathbb{N}$ satisfies $A(r+1) \subseteq B(0, \epsilon) \subseteq A(r)$. By Lemma 7.8 the maps from $\widehat{A}(r)$ onto maximal pre-images of the decomposition of $A(s)$ form a countable family of contractions accumulating only the critical point and whose contraction ratios satisfy

$$
0<g_{s, r} \leq \frac{\left|\widehat{A}^{s}\right|}{\left|\widehat{A}^{r}\right|} \leq c 2^{-\frac{1}{2}\left(N_{s}-N_{r}\right)+2 \rho s} \leq c 2^{\bar{M} r-(1-2 \rho) s}<1
$$

since the decomposition of $A(s)$ only contains maximal pre-images of $A(r)$ if $r \leq \omega s \ll s$. We can thus apply Proposition 6.1 , so $\operatorname{dim}_{H}\left(\widetilde{\mathcal{E}}_{\epsilon}\right) \leq d$ for every $d \in \mathbb{R}$ with $\sum_{s, r \in \mathbb{N}} \widehat{\nu}(s, r) g_{s, r}^{d} \leq 1$. As

$$
\sum_{s, r \in \mathbb{N}} \widehat{\nu}(s, r) g_{s, r}^{d} \leq \sum_{\omega s \geq r} c 2^{\left(\frac{2 \log (r / s) \log \left(c \omega \bar{M} s^{2}\right)}{s \log \omega}-(1-2 \rho-\bar{M} \omega) d\right) s} .
$$

and as $\omega \ll \bar{M}^{-1}$ the exponent is negative and the sum is bounded by

$$
\sum_{s, r \in \mathbb{N}} \widehat{\nu}(s, r) g_{s, r}^{d} \leq c 2^{2 \log \left(c \bar{M} \omega^{-1} r^{2}\right)-(1-2 \rho-\bar{M} \omega) d \omega^{-1} r} .
$$

Thus

$$
\operatorname{dim}_{H}\left(\widetilde{\mathcal{E}}_{\epsilon}\right) \leq d_{0}:=\frac{c \log \left(c \bar{M} \omega^{-1} r^{2}\right)}{r\left(\omega^{-1}-\left(\bar{M}^{2}+2\right) / \bar{M}\right)} \leq \frac{c \log r}{r} .
$$

As $A(r+1) \subseteq B(0, \epsilon) \subseteq A(r)$, we have $r \leq\left|\log _{2} \epsilon\right| \leq \bar{M} r+\frac{1}{2} M$ as in Proposition 5.1. As $\epsilon<\left|\widetilde{\alpha}^{M-1}\right| \leq c 2^{-M}$ by Proposition 5.2, we have $\operatorname{dim}_{H}\left(\widetilde{\mathcal{E}}_{\epsilon}\right) \leq c \log _{2}\left|\log _{2} \epsilon\right| /\left|\log _{2} \epsilon\right|$.

\section{BIBLIOGRAPHY}

[1] Avila (A.), Lyubich (M.) \& De Melo (W.) - Regular or stochastic dynamics in real analytic families of unimodal maps, Preprint, 2001.

[2] Avila (A.) \& Moreira (C.G.) - Statistical properties of unimodal maps: smooth families with negative Schwarzian derivative, in Geometric Methods in Dynamics (I) (de Melo (W.), Viana (M.) \& Yoccoz (J.-C.), eds.), Astérisque, vol. 286, Soc. Math. France, Paris, 2003, pp. 81-118.

[3] _ Statistical properties of unimodal maps: the quadratic family, to appear in Ann. of Math., 2003.

[4] Benedicks (M.) \& CARleson (L.) - On iterations of $1-a x^{2}$ on $(-1,1)$, Ann. of Math. (2), t. 122 (1985), no. 1, pp. 1-25.

[5] — The dynamics of the Hénon map, Ann. of Math. (2), t. 133 (1991), no. 1, pp. $73-169$.

BULLETIN DE LA SOCIÉTÉ MATHÉMATIQUE DE FRANCE 
[6] Bowen (R.) - Equilibrium states, Lect. Notes in Math., vol. 470, Springer Verlag, 1975.

[7] FAlconer (K.) - Fractal Geometry; Mathematical Foundations and Applications, John Wiley, 1990.

[8] Graczyk (J.) \& ŚwiA̧tek (G.) - Generic hyperbolicity in the logistic family, Ann. of Math. (2), t. 146 (1997), no. 1, pp. 1-52.

[9] Jakobson (M. V.) - Absolutely continuous invariant measures for oneparameter families of one-dimensional maps, Comm. Math. Phys., t. 81 (1981), no. 1, pp. 39-88.

[10] Luzzatto (S.) - Bounded recurrence of critical points and Jakobson's theorem, in The Mandelbrot set, theme and variations, Cambridge Univ. Press, Cambridge, 2000, pp. 173-210.

[11] Lyubich (M.) - Dynamics of quadratic polynomials. I, II, Acta Math., t. 178 (1997), no. 2, pp. 185-247, 247-297.

[12] DE Melo (W.) \& VAN Strien (S.) - One-dimensional dynamics, Springer-Verlag, Berlin, 1993.

[13] RÉNyi (A.) - Representations for real numbers and their ergodic properties, Acta Math. Acad. Sci. Hungar, t. 8 (1957), pp. 477-493.

[14] Rychlik (M. R.) - Another proof of Jakobson's theorem and related results, Ergodic Theory Dynamical Systems, t. 8 (1988), no. 1, pp. 93-109.

[15] Senti (S.) - Dimension de Hausdorff de l'ensemble exceptionnel dans le théorème de Jakobson, Ph.D. Thesis, Université de Paris-Sud, 2000, available at http://www.math.psu.edu/senti.

[16] Tsujil (M.) - A proof of Benedicks-Carleson-Jacobson theorem, Tokyo J. Math., t. 16 (1993), no. 2, pp. 295-310.

[17] Yoccoz (J.-C.) - Jakobson's Theorem, Manuscript of Course at Collège de France, 1997. 